\title{
A prospective cohort study evaluating the effectiveness of cardiac arrest and resternotomy simulation training of the post-cardiac surgery recovery team
}

\author{
Andrew G. Keogan, ${ }^{a, *}$ Richard M. Maier Jr. ${ }^{a}$ and Molly M. Hagen ${ }^{b}$ \\ ${ }^{a}$ Reno School of Medicine, University of Nevada, Reno, NV 89557, USA; ${ }^{b}$ School of Community Health Sciences, University of \\ Nevada, Reno, NV 89557, USA
}

${ }^{*}$ Corresponding author at: 1664 N Virginia St, Reno, NV 89557, USA. Email: akeogan@med.unr.edu

Date accepted for publication: 23 September 2021

\section{Abstract}

Objective: The management of cardiac arrest in after cardiac surgery requires resuscitative efforts that deviate from the established American Heart Association Advanced Cardiac Life Support guidelines (ACLS) guidelines. The Society of Thoracic Surgeons (STS) and the European Association of Cardiothoracic Surgery have established guidelines for managing cardiac arrest and resternotomy in these patients and recommend regular practice by the multidisciplinary recovery team. This study, conducted at a single institution, aimed to identify areas of deficiency in knowledge of STS guidelines and evaluate the effects of simulation on comfort with the procedure and perceived value of training. Methods: A before and after study was performed whereby participants were given hands-on resternotomy training and completed pre- and post-training surveys that assessed knowledge of the STS protocol, comfort level, and value perception. The training, simulation, and survey were conducted in a clinical simulation laboratory. Study participants included registered nurses, attending physicians, resident physicians, pharmacists, and respiratory therapists. The training consisted of a lecture followed by a high-fidelity simulation of a cardiac arrest leading to bedside surgical exploration utilizing a resternotomy manikin. Results: Participants' baseline scores of knowledge of STS guidelines averaged 53.3\%. This average increased to $81.3 \%$ after training, with improvement on four of the six knowledge questions achieving statistical significance $(P<0.001$ in all cases). Participant comfort with the STS protocol and the resternotomy procedure increased significantly (both $P<0.0001)$, as well as value perceptions of the simulation training $(P<$ $0.03)$ and utilizing a resternotomy capable manikin $(P<0.02)$. Conclusions: Participants' inadequate knowledge of STS guidelines suggests that delivering education to all post-cardiac surgery team members would be beneficial and is desired by clinicians. High-fidelity resternotomy simulation has favorable effects on participant comfort and their perceived value of training and can be made affordable by modifying widely available supplies.

Keywords: ICU resternotomy; cardiac surgery simulation; resternotomy manikin; post-cardiac surgery cardiac arrest; multidisciplinary simulation

\section{Introduction}

Cardiac surgeries are increasingly standard procedures performed in American hospitals; more than 400,000 procedures occur each year. ${ }^{1}$ Post-surgical cardiac arrest among these patients occurs in $0.7 \%-8 \%$ of cases. ${ }^{2}$ With timely and appropriate treatment, post-cardiac surgery cardiac arrest patients have better outcomes compared with cardiac arrest patients from other settings; $50 \%$ survive to discharge. ${ }^{3-6}$ Prompt, protocol-driven resuscitation by specially trained staff after cardiac arrest in this patient population reduces time to resternotomy, improves survival, and reduces the complications of bedside re-exploration of the surgical site. ${ }^{4,7,8}$ Substantial differences exist between the Society of Thoracic Surgeons (STS) protocol for cardiac arrest in cardiac surgical patients and standard American College of Cardiology (ACC)/American Heart Association (AHA) Advanced Cardiac Life Support guidelines (ACLS). As a result, advanced training is recommended for teams responsible for the intensive care unit (ICU) treatment of the cardiac surgical patient to adequately prepare for the necessary interventions to optimize survival during a postsurgical cardiac arrest. $^{2}$

Considering this recommendation, our 808-bed regional referral facility implemented a classroom training and 
high-fidelity simulation course outlining STS arrest guidelines and bedside resternotomy procedure. Using a before and after survey design, we sought to identify areas of knowledge deficiency and define how simulation of the resternotomy technique affected team members' comfort levels with participation in this emergent procedure and the perceived value of this training. We hypothesized that ICU team members would initially be unfamiliar and uncomfortable with STS guidelines and that our multidisciplinary training would increase knowledge and confidence levels with emergent bedside resternotomy. Our study results can benefit other cardiac surgery recovery teams by highlighting educational opportunities and the value of implementing a high-fidelity resternotomy simulation.

\section{Materials and methods}

Study type and study design

This prospective cohort study evaluated the effectiveness of cardiac arrest and resternotomy simulation training of the post-cardiac surgery recovery team.

Study PICO (population, intervention, control, and outcomes)

\section{Population (P)}

Study participants were post-cardiac surgery team members, including registered nurses, attending physicians, resident physicians, pharmacists, and respiratory therapists.

\section{Intervention (I)}

The training consisted of a lecture followed by a high-fidelity simulation of a cardiac arrest leading to bedside surgical exploration utilizing a resternotomy manikin, whereby participants were given hands-on resternotomy training. The training consisted of a classroom presentation and included a review of the post-surgical cardiac arrest protocol, equipment, and roles involved with bedside resternotomy. A case format involving a simulated patient who suffers an arrest immediately after cardiac surgery was used with the simulation outcome dependent on correctly using multiple pathways of the STS treatment protocols in expert consensus statement on patients who arrest after cardiac surgery.

\section{Comparing (C)}

A before and after study whereby participants were given hands-on resternotomy training and completed pre- and post-training surveys.

\section{Outcome measure $(\mathrm{O})$}

Assessing knowledge of the STS protocol, comfort level, and value perception. We administered identical anonymous online questionnaires to all participants before and after the training/simulation sequence to evaluate practitioners' knowledge, comfort, and attitudes on resternotomy at baseline and after the training.

\section{Setting}

The training, simulation, and survey were conducted in a general clinical simulation laboratory. Recovery team members included cardiac ICU-trained registered nurses, critical care fellowship-trained attending physicians, internal medicine residents, pharmacists, and respiratory therapists. The ICU is a 38-bed combined medical cardiac ICU/cardiac surgery unit that sees between 300 and 400 cardiac surgery cases per year. Surgical patients receive all their post-operative care until discharge in this unit. Our facility's postoperative care is co-managed by the cardiothoracic surgery and critical care services. There is no cardiothoracic surgeon in house overnight. Emergent resternotomy responsibilities are covered by the in-hospital trauma surgeon or critical care physician. Registered nurses and advanced practice nurses are only permitted to assist in the event of a resternotomy. Our training followed a newly adopted hospital protocol that provided roles and procedural steps for the resuscitation and resternotomy of patients who arrest after cardiac surgery and reflects the recommendations set by the STS expert consensus statement on patients who arrest after cardiac surgery. ${ }^{2}$ The protocol is intended for patients who have undergone cardiac surgery within 10 days and was performed in the cardiac surgical unit.

\section{Study protocol}

After a classroom presentation, the team was introduced to the simulation room, manikin, and equipment. Enrollment in each session was limited to ensure participants had a role defined by the hospital protocol. An initial walkthrough of the resuscitation protocol allowed participants to practice the procedures. After this orientation, facilitators presented the patient scenario, and the simulation commenced. This scenario described a patient arriving from the operating room status post aortic valve replacement with a median sternotomy who rapidly develops substantial bleeding and progresses to cardiac arrest. The resuscitation team consisted of the six key roles defined by the STS, with the addition of a second external cardiac massage nurse. ${ }^{2}$ For resternotomy, two nurses transition roles to scrub nurse and assistant, and the intensivist opens the chest (Fig. 1). Nurses were cross-trained on all roles except the physician role. An intensivist and nurse educator facilitated the simulation. After the simulation, the facilitators debriefed the team. The simulation utilized two manikins: a SimMan3G (Laerdal Medical; Stavanger, Norway) for the initial cardiac arrest portion and a modified Little Anne CPR manikin for the resternotomy procedure (Fig. 2). The SimMan3G was 


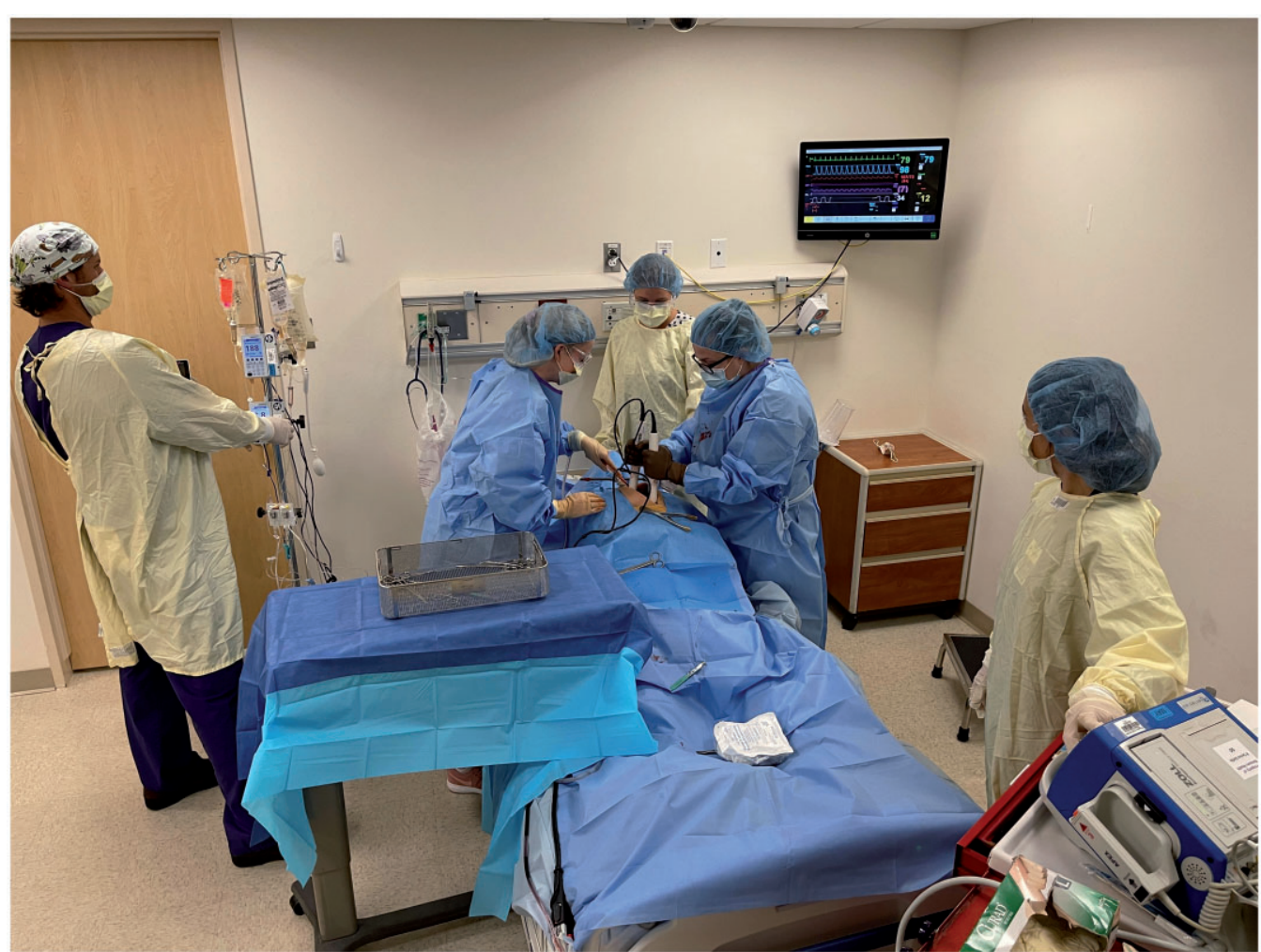

Figure 1. Resuscitation team performing simulated bedside resternotomy in the laboratory.

prepared with patient monitoring and live feed data, endotracheal tube, mediastinal drains, epicardial wires, and intravenous lines with pumps. When the resternotomy portion of the simulation occurred, the resternotomy manikin was laid on the full-body SimMan3G manikin and subsequently draped. The resternotomy manikin was modified with spring-loaded door hinges to allow resternotomy, including cutaneous suture cutting, sternal wire removal, sternal retractor placement and actuation, sterile suction, evacuation of pericardial hematoma, open cardiac massage, open chest defibrillation, and hemorrhage control of the aortic cannulation site. Additional equipment included mock medications, a resternotomy cart with surgical instruments, a code cart with a defibrillator, and bedside tables. Appropriate sterile supplies such as gowns, gloves, drapes, etc. were also available.

\section{Questionnaire}

The questionnaire contained three components: participant characteristics, STS knowledge, and attitudes.

\section{Ethics approval}

An institutional review board approved the project as exempt and classified the research as social behavior on June 10, 2019. The IRBnet identification number is 1416368-1.

\section{Data collection}

Data were collected over eight training sessions on three separate days between May 2019 and December 2019.

(1) We collected data on participant characteristics using four questions about the participant's discipline and experience levels.

(2) The second component consisted of four multiple-choice questions (MCQ), one true or false question, and one select-all-that-apply question. These objective questions tested knowledge of crucial resuscitation principles for cardiac surgery patients taken directly from STS consensus/hospital policy.

(3) The third section evaluated the participant's confidence and comfort with resternotomy and the perceived value of simulation training, training frequency, and equipment use. These subjective questions were graded on a 5-point Likert scale. The answers to the knowledge questions were not reviewed during the training. However, the topics were discussed in the context of the arrest protocol during the training.

\section{Statistical methods}

We calculated knowledge scores as the percentage of the knowledge questions answered correctly at each time point. We could not link an individual's pre- and posttraining surveys because we did not collect personally identifiable information. Consequently, we used a two-sample $t$ test to compare the overall pre- and post-training test scores 


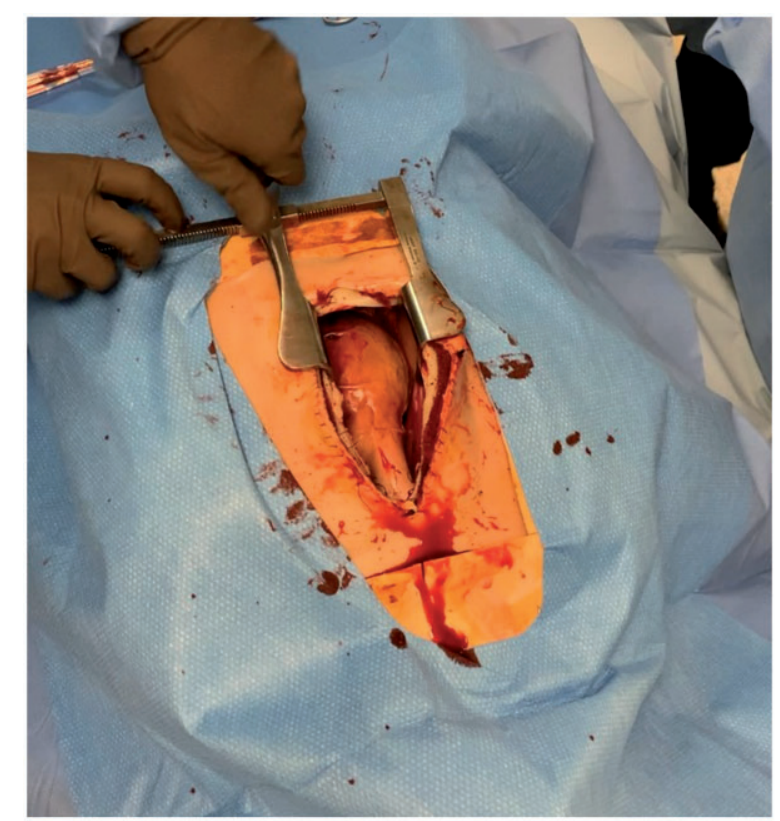

Figure 2. Modified CPR manikin allows for resternotomy, including incision reopening, cutting sternal wires, and retraction of the sternum.

and chi-squared tests to evaluate pre- and post-training changes in the proportion of people who answered each question correctly. We used linear regressions to examine relationships between participant discipline, experience level, and previous performance of a resternotomy on baseline test scores. We characterized participant experiences and perspectives using six subjective questions. The subjective questions were measured on 5-point Likert scales with higher values corresponding to positive/favorable emotions and lower values corresponding to negative/unfavorable emotions. Differences in subjective measures before and after training were calculated using numerical equivalents and tested using Wilcoxon rank-sum tests (a non-parametric two-sample $t$ test equivalent). For all analyses, we used R Studio, version 3.6.1 (R Foundation for Statistical Computing; Vienna, Austria) and assessed statistical significance at $\alpha=0.05$. Summary statistics are presented as means \pm standard error (SE).

\section{Results}

Fifty-two individuals completed 103 surveys between eight training sessions ( $n=51$ pre-training and $n=52$ posttraining). The overall test scores for participants before they received the training was $53.3 \% \pm 3.1 \%$. Overall, test scores for participants increased by $52.5 \%$ after training to $81.3 \% \pm 1.6 \%$.

\section{Demographics}

Thirty-three of 51 participants had less than 5 years of experience in their field. Only $15.7 \%$ of participants had received resternotomy training before the current event, and previous training was not related to pre-training test scores $(F=0.010, P=0.525$; Table 1$)$. The sample was primarily composed of registered nurses (RN) (66.7\%) followed by medical doctors (MD)/doctors of osteopathic medicine (DO) (17.6\%), pharmacists (11.8\%), and respiratory therapists (RT) (3.9\%). Because only two respiratory therapists were sampled, they were excluded from tests of discipline on overall test scores; doctors, nurses, and pharmacists did not show significantly different test scores overall $(F=$ 0.491, $P=0.615)$, although nurses had slightly lower scores than doctors $(P=0.344$; Table 1$)$. Neither years of experience in their respective discipline nor previous experience conducting a bedside resternotomy were related to participant pre-training test scores (in both cases $P \geqslant 0.428$; Table 1).

\section{STS guideline test questions}

Participant's scores improved significantly from before to after training for four of the six knowledge questions. All four of these questions were multiple-choice questions with improvements demonstrating $P=0.0001$ or less (Table 2). The remaining two questions did not show significant change. The true or false question was answered correctly by most participants (average $86.3 \%$ correct) at baseline. The final question, select-all-that-apply, displayed the lowest correct percentage and decreased in post-training scores from $23.5 \%$ to $19.2 \%$ (Table 2).

\section{Subjective questions}

The subjective portion of the questionnaire began by asking, "How often should your discipline (RN/RT/MD/DO/ Pharmacists) train for cardiac arrest and emergent bedside resternotomy scenarios?" Before the training, 15 individuals said more than once a year, 30 individuals said once a year, five individuals said once every 2 years, and one individual said once is sufficient. After the training, 23 individuals said resternotomy training should be administered more than once a year, 25 individuals said once a year, three individuals said once every 2 years, and one individual said once is sufficient.

Both subjective questions regarding comfort (related to the guidelines and participation in a resternotomy) showed significant increases in scores after training and simulation (both $P \leqslant 0.0001$; Table 3). Subjective assessment of confidence levels identifying indications for an emergent resternotomy showed significant increases after the simulation $(P \leqslant 0.0001)$. However, these subjective data stand in 
Table 1. Participant demographics and relationships to pre-training test scores

\begin{tabular}{|c|c|c|c|c|c|c|c|c|}
\hline Variable & Levels & $n$ & $\%$ & Mean score $\pm \mathrm{SE}$ & $\beta$ & $P$ value & $F$ & $P$ value \\
\hline \multirow[t]{4}{*}{ Discipline } & $\mathrm{RN}$ & 34 & 66.7 & $51.5 \pm 3.6$ & -8.0 & 0.344 & \multirow[t]{4}{*}{0.491} & \multirow[t]{4}{*}{0.615} \\
\hline & $\mathrm{MD} / \mathrm{DO}$ & 9 & 17.6 & $59.4 \pm 6.9$ & Ref & Ref & & \\
\hline & $\mathrm{RT}^{*}$ & 2 & 3.9 & $50.0 \pm 17.0$ & $\mathrm{n} / \mathrm{a}$ & $\mathrm{n} / \mathrm{a}$ & & \\
\hline & Pharmacy & 6 & 11.8 & $55.7 \pm 12.6$ & -3.8 & 0.749 & & \\
\hline \multirow[t]{4}{*}{ Years of experience } & $<2$ & 7 & 13.7 & $45.3 \pm 11.8$ & Ref & Ref & \multirow[t]{4}{*}{0.428} & \multirow[t]{4}{*}{0.734} \\
\hline & $2-5$ & 26 & 51.0 & $54.5 \pm 4.4$ & 9.3 & 0.333 & & \\
\hline & $5-10$ & 14 & 27.5 & $56.0 \pm 4.5$ & 10.7 & 0.303 & & \\
\hline & $>10$ & 4 & 7.8 & $50.0 \pm 9.8$ & 4.7 & 0.737 & & \\
\hline \multirow[t]{2}{*}{ Previous training } & Yes & 8 & 15.7 & $54.0 \pm 9.8$ & 0.8 & 0.924 & \multirow[t]{2}{*}{0.010} & \multirow[t]{2}{*}{0.924} \\
\hline & No & 43 & 84.3 & $53.2 \pm 3.2$ & Ref & Ref & & \\
\hline \multirow[t]{3}{*}{ Previous experience } & 0 & 33 & 64.7 & $53.1 \pm 3.9$ & Ref & Ref & \multirow[t]{3}{*}{0.654} & \multirow[t]{3}{*}{0.525} \\
\hline & 1 & 13 & 25.5 & $50.0 \pm 5.7$ & -3.1 & 0.667 & & \\
\hline & 2 & 5 & 9.8 & $63.2 \pm 9.7$ & 10.1 & 0.345 & & \\
\hline
\end{tabular}

DO, doctor of osteopathic medicine; MD, medical doctor; RN, registered nurse; RT, respiratory therapist; SE, standard error.

* RT was not included in linear regression due to insufficient sample size.

Table 2. Percentage of participants answering questions correctly before and after training

\begin{tabular}{|c|c|c|c|c|}
\hline Question & Answer & Pre-training, $n / N(\%)$ & Post-training, $n / N(\%)$ & $P$ value \\
\hline $\begin{array}{l}\text { According to the Society of Thoracic Surgeons (STS), how many } \\
\text { defibrillations (DC shocks) should be performed before } \\
\text { beginning CPR in a cardiac surgery patient with a presenting } \\
\text { rhythm of ventricular fibrillation? }\end{array}$ & Three & $29 / 51(56.9)$ & $52 / 52(100)$ & $<0.0001$ \\
\hline $\begin{array}{l}\text { Although the STS does not recommend epinephrine for cardiac } \\
\text { arrest in cardiac surgery patients, they do acknowledge its } \\
\text { utility. They suggest its use be restricted to clinicians experi- } \\
\text { enced with its use in cardiac surgery. What is the epinephrine } \\
\text { dose range the STS specifies? }\end{array}$ & $50-300 \mu \mathrm{g}$ & $30 / 51(58.8)$ & $51 / 52(98.1)$ & $<0.0001$ \\
\hline $\begin{array}{l}\text { In a cardiac surgery patient presenting with asystole, what is the } \\
\text { STS recommended first-line treatment? }\end{array}$ & Epicardial pacing & $24 / 51(47.1)$ & $44 / 52(84.6)$ & 0.0001 \\
\hline $\begin{array}{l}\text { True or false. According to the STS, internal cardiac massage is } \\
\text { superior to chest compressions in the patient with recent } \\
\text { sternotomy requiring resuscitative efforts beyond } 5 \text { minutes }\end{array}$ & True & $44 / 51(86.3)$ & $47 / 52(90.4)$ & 0.7317 \\
\hline $\begin{array}{l}\text { When chest compressions or internal cardiac massage is } \\
\text { required, what is the target systolic pressure recommended by } \\
\text { the STS? }\end{array}$ & $>60 \mathrm{~mm} \mathrm{Hg}$ & $24 / 51(47.1)$ & $50 / 52(96.2)$ & $<0.0001$ \\
\hline $\begin{array}{l}\text { Which complications are indications for emergent bedside rest- } \\
\text { ernotomy? Select all that apply }\end{array}$ & $\begin{array}{l}\text { Cardiac tamponade, } \\
\text { patient POD 0-10 } \\
\text { in cardiac arrest, } \\
\text { and post-operative } \\
\text { hemorrhage }\end{array}$ & $12 / 51(23.5)$ & $10 / 52(19.2)$ & 0.7705 \\
\hline
\end{tabular}

Statistically significant improvements are shown in bold.

contrast to the objective question scores regarding the same topic. The perceived importance of resternotomy training also increased after the simulation $(P=0.0298$; Table 3$)$. Although participants rated both the value of supplies and the resternotomy manikin higher on post-training surveys, they did so with slightly more preference for the resternotomy manikin, which reached statistical significance at $P=$ 0.0192 compared with supplies at $P=0.0852$. Participants rated using supplies and equipment highly on their pre-training surveys at an average of $4.51 \pm 0.11$ on a 5-point Likert scale.

\section{Discussion}

In this before and after survey, we sought to identify areas of knowledge deficiency and found an inadequate understanding of appropriate interventions for cardiac arrest after cardiac surgery across all disciplines and experience 
Table 3. Summary statistics (mean \pm SE) for participant evaluation of the training based on a five-point Likert scale

\begin{tabular}{|c|c|c|c|}
\hline Question & Pre-training & Post-training & $P$ value \\
\hline $\begin{array}{l}\text { Please indicate your comfort level with the resternotomy policy implemented in 2019, } \\
\text { which is based on recommendations by the Society of Thoracic Surgeons (STS) }\end{array}$ & $2.11 \pm 0.15$ & $3.65 \pm 0.11$ & $<0.0001$ \\
\hline $\begin{array}{l}\text { Please rate your confidence level in identifying patient complications that are indications } \\
\text { for emergent resternotomy }\end{array}$ & $2.76 \pm 0.18$ & $3.98 \pm 0.10$ & $<0.0001$ \\
\hline $\begin{array}{l}\text { Please indicate your comfort level with participating in an emergent resternotomy as a } \\
\text { primary team member in your discipline }\end{array}$ & $2.31 \pm 0.15$ & $3.71 \pm 0.10$ & $<0.0001$ \\
\hline $\begin{array}{l}\text { How important is emergent bedside sternotomy training for the cardiac surgical unit } \\
\text { (CSU) multidisciplinary team? }\end{array}$ & $4.61 \pm 0.12$ & $4.87 \pm 0.07$ & 0.0298 \\
\hline $\begin{array}{l}\text { Please rate the value of training for resternotomy with supplies and equipment such as } \\
\text { sterile drapes, gowns, gloves, betadine, surgical tray, instruments, internal defib pads, } \\
\text { etc. }\end{array}$ & $4.51 \pm 0.11$ & $4.75 \pm 0.08$ & 0.0852 \\
\hline $\begin{array}{l}\text { Please rate the training value of performing a simulated bedside resternotomy with a } \\
\text { manikin specific to the procedure }\end{array}$ & $4.49 \pm 0.07$ & $4.79 \pm 0.10$ & 0.0192 \\
\hline
\end{tabular}

Likert scale: 5, very comfortable/confident/important; 3, neutral; and 1, very uncomfortable/not confident/not valuable/not important. Statistically significant changes are shown in bold.

levels. We tested critical components of the STS arrest algorithm, all of which are deviations from AHA ACLS cardiac arrest guidelines. Some guidelines are in stark contrast, such as pacing asystole or avoiding the routine use of epinephrine, which may lead to hypertension and subsequent bleeding. ${ }^{2}$ With pre-training test results below $60 \%$ in every discipline, we identified this as an important educational opportunity for all team members. We acknowledge the sample populations from disciplines other than nursing were small, but the results suggest this education would be beneficial for all roles of the resuscitation team to avoid misunderstandings and improve team dynamics during arrest scenarios. Moreover, participants from all disciplines indicated a desire for regular training.

Further breakdown of the six test questions revealed that only the multiple-choice questions achieved statistical significance. The select-all-that-apply question concerning indications for resternotomy was answered correctly by $23.5 \%$ before training and $19.2 \%$ after training. This result contrasts with the post-training question regarding confidence identifying indications for emergent resternotomy, which revealed a significant increase. These conflicting results could indicate a weak correlation of confidence levels with understanding, unclear answer choices, or lack of emphasis during training. Analysis revealed 33 participants identified "POD 0-10 in cardiac arrest" as an indication on the pre-test, while only 26 did so correctly on the post-test. This result suggests a need for increased emphasis on all the indications for resternotomy. However, 52 of 52 participants did correctly selected tamponade and hemorrhage as indications on the post-test. The overall increase in test scores from $53.3 \%$ to $81.3 \%$ illustrates the effectiveness of the classroom training and simulation on improving knowledge of post-cardiac surgery cardiac arrest guidelines.

Another primary focus of the survey was to assess the effect of resternotomy simulation on the participants' comfort and their perceived value of the training. Previous research has indicated resternotomy simulation as an effective means of improving time to chest reopen and confidence performing the procedure. ${ }^{7,9}$ Building on this evidence, we found participants overwhelmingly reported increased comfort levels with both the guidelines and performing the procedure (both $P<0.0001$ ). We found training value suggested in several aspects of the study, including 48 of 52 respondents preferring a simulation training schedule once a year or more. Also, ratings of performing the simulation with a resternotomy-specific manikin increased after the training $(P<0.02)$. The use of sterile supplies and equipment was rated highly both before and after (4.51 and 4.75, respectively). We hope these results will contribute to the costbenefit discussion of performing the simulation with consumable supplies (gowns, gloves, drapes, etc.). In addition, the resternotomy manikin represents a one-time investment that facilities can significantly reduce in cost by modifying a CPR manikin (hardware to modify cost less than US\$100) similar to our task trainer.

\section{Limitations}

We acknowledge several limitations of our study, including the relative inexperience of the participating staff and the small sample sizes from MD/DO, pharmacy, and RT. Also, the facility could be considered a low-volume cardiac surgery center with likely lower rates of resternotomy that may have an impact on team member experience and limit the 
evaluation of simulation training on bedside procedural performance.

\section{Conclusions}

With regular training aimed at improving adherence to STS guidelines and proficiency with rapid resternotomy, it may be possible to improve patient outcomes. ${ }^{7}$ Since the development of this training, the resuscitation team has performed two emergent resternotomies, both with favorable patient outcomes. Feedback from the resuscitation team received during debriefing was remarkable for effective coordination, efficiency, and performance. As a result of this study and feedback from real-life events, our institution has made yearly simulation training mandatory for all nursing staff caring for cardiac surgery patients and recommended for physicians, pharmacists, and respiratory therapists. We hope this study's positive results surrounding simulation and the identified knowledge deficit may encourage cardiac surgery recovery teams to devote the resources needed to implement post-cardiac surgery cardiac arrest resternotomy simulation training.

\section{Conflict of interest}

The authors have no conflict of interest to declare.

\section{Acknowledgement}

We want to acknowledge the Renown Regional Medical Center cardiac surgery recovery team, including Stephanie Caffery, BSN, RN, CCRN, Liz Hassler, BSN, RN, CCRN, and Penny Burke, BSN, RN, for facilitating the simulation as well as their contributions to the development of classroom and simulation content.

\section{References}

1. American Heart Association Statistics Committee and Stroke Statistics Subcommittee. Heart disease and stroke statistics2010 update: a report from the American Heart Association.
Circulation 2010; 121(7): e46-e215. https://doi.org/10. 1161/CIRCULATIONAHA.109.192667.

2. Society of Thoracic Surgeons Task Force on Resuscitation After Cardiac Surgery. The Society of Thoracic Surgeons expert consensus for the resuscitation of patients who arrest after cardiac surgery. Ann Thorac Surg 2017; 103: 1005-1020. https://doi.org/10.1016/j.athoracsur.2016.10.033.

3. Charalambous CP, Zipitis CS, Keenan DJ. Chest re-exploration in the intensive care unit after cardiac surgery: a safe alternative to returning to the operating theater. Ann Thorac Surg 2006; 81: 191-194. https://doi.org/10.1016/j. athoracsur.2005.06.024

4. Maccaroni MF, Watson ND, Gaage DL. Managing cardiac arrest after cardiac surgery: the impact of a five year evolving resternotomy policy and a review of the literature. Analg Resusc Curr Res 2013; S1: 1-7. https://doi.org/10. 4172/2324-903X.S1-008.

5. Mackay JH, Powell SJ, Osgathorp J, Rozario CJ. Six-year prospective audit of chest reopening after cardiac arrest. Eur J Cardiothorac Surg 2002; 22: 421-425. https://doi.org/10. 1016/S1010-7940(02)00294-4.

6. Pottle A, Bullock I, Thomas J, Scott L. Survival to discharge following open chest cardiac compression (OCCC). A 4-year retrospective audit in a cardiothoracic specialist centre - Royal Brompton and Harefield NHS Trust, United Kingdom. Resuscitation 2002; 52: 269-272. https://doi.org/10. 1016/S0300-9572(01)00479-8.

7. Dunning J, Nandi J, Ariffin S, Jerstice J, Danitsch D, Levine A. The cardiac surgery advanced life support course (CALS): delivering significant improvements in emergency cardiothoracic care. Ann Thorac Surg 2006; 81: 1767-1772. https://doi.org/10.1016/j.athoracsur.2005.12.012.

8. Reddy HG, Shih T, Englesbe MJ, Shannon FL, Theurer PF, Herbert MA, et al. Analyzing "failure to rescue": is this an opportunity for outcome improvement in cardiac surgery? Ann Thorac Surg 2013; 95: 1976-1981. https://doi.org/10. 1016/j.athoracsur.2013.03.027.

9. Nunnink L, Welsh AM, Abbey M, Buschel C. In situ simulation-based team training for post-cardiac surgical emergency chest reopen in the intensive care unit. Anaesth Intensive Care 2009; 37: 74-78. https://doi.org/10.1177/0310057X0903700109. 